\title{
Experimental and numerical studies on super-cavitating flow of axisymmetric cavitators
}

\author{
Byoung-Kwon Ahn, Chang-Sup Lee and Hyoung-Tae Kim
}

Department of Naval Architecture and Ocean Engineering, College of Engineering, Chungnam National University, Korea

\begin{abstract}
Recently underwater systems moving at high speed such as a super-cavitating torpedo have been studied for their practical advantage of the dramatic drag reduction. In this study we are focusing our attention on super-cavitating flows around axisymmetric cavitators. A numerical method based on inviscid flow is developed and the results for several shapes of the cavitator are presented. First using a potential based boundary element method, we find the shape of the cavitator yielding a sufficiently large enough cavity to surround the body. Second, numerical predictions of supercavity are validated by comparing with experimental observations carried out in a high speed cavitation tunnel at Chungnam National University (CNU CT).
\end{abstract}

KEY WORDS: Super-cavitation; Cavitator; Axisymmetric cavitating flow; Boundary value problem.

\section{INTRODUCTION}

If the speed of the submerged body is sufficiently high, and the local pressure drops to the vapor pressure of the fluid then cavitation occurs on the body surface. As the speed goes up further and the cavity is large enough to cover the entire body then it is called supercavitation. The drag force acting on a supercavitating body is very low compared with a fully wetted body because the friction drag in the air is nearly 1,000 times less in that of the water. One tries to apply the main advantage of the supercavitation, drag reduction, to high speed underwater vehicles like a torpedo.

In this study, we focus our attention on a cavitator which initiates and generates the supercavity. We firstly consider various symmetric wedges and predict cavity length using a potential based boundary element method. Predicted results are validated by comparing with an analytic solution for the supercavity flow around the symmetric body (Newman, 1977). We carried out two dimensional experiments in a high speed cavitation tunnel and observed the supercavity occurring on the symmetric wedge. Numerical results are compared with experimental measurements. The present method is capable of predicting the supercavity sufficiently well and it can be a useful tool to design a super-cavitator

\section{NUMERICAL FORMULATION}

We assume that the flow is inviscid, incompressible and

Corresponding author: Byoung-Kwon Ahn

e-mail: bkahn@,cnu.ac.kr irrotational. The total velocity $(\bar{V})$ is expressed in terms of the total velocity potential $(\Phi)$, which takes the form:

$$
\bar{V}=\nabla \Phi=\nabla\left(U_{\infty} \cdot \bar{x}+\phi\right)
$$

where $U_{\infty}$ is the on-coming velocity, $\bar{x}$ is the position vector and $\phi$ is the perturbed velocity potential.

The assumed flow should be satisfied with conservation of the mass and it yields the Laplace equation as a governing equation:

$\nabla \cdot \bar{V}=\nabla^{2} \Phi=0$

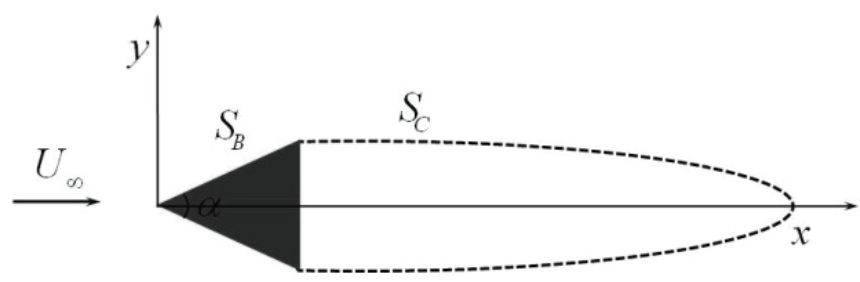

Fig. 1 Symmetric body (cavitator) and cavity surface.

We consider two dimensional symmetric body showing in Fig. 1. Here $S_{B}$ and $S_{C}$ denote body and cavity surface respectively. Boundary conditions are applied and the flow is uniquely defined. Firstly, the flow is not perturbed at infinity of the assumed flow and also it does not penetrate the body surface, which states in Equation (3) and (4).

$\lim _{r \rightarrow \infty} \nabla \Phi=U_{\infty}$ 
$\nabla \Phi \cdot \bar{n}=\bar{n} \cdot U_{\infty}$

where $\bar{n}$ is the outward unit normal vector to the boundary. Second, kinematic and dynamic boundary conditions are applied to the cavity surface as follows.

$\frac{D f(x, y)}{D t}=0$

$p=p_{v}$

where $f(x, y)$ is a cavity surface and $p_{v}$ is the vapor pressure of fluid, the pressure inside the supercavity.

In addition, we apply that the cavity detached from the body is naturally closed at the end of the cavity. A closed body with constant pressure around its surface does not exist in an exact potential flow so that one should apply a proper termination condition at the end of the cavity. Several termination models have been suggested representatively by Kreise (1946), Wu (1972) and Lee (1989). Lee suggested the linear termination model stating that the cavity shape is dependent only on the source strength and the sum of strengths of the sources should be zero to make the surface close. To apply the linear model, it is necessary to present the stagnation point at the after end of the cavity as in the case of real flow around a thick round trailing edge. This is a dominant factor in symmetric flow problem. In this flow, the stagnation point is coincident with the after end point of the cavity. Considering that the velocity on the cavity surface is constant except at the end of the cavity, one may apply that the cavity shape is elliptic at the end of it. It is applied in the present study and the nonlinear cavity shape may therefore be found with minimum number of iterations. Here, the cavity shape is initially assumed to be elliptic.

We get a relation between the pressure and the velocity from the Bernoulli's equation:

$C_{P} \equiv \frac{p-p_{\infty}}{1 / 2 \rho U_{\infty}^{2}}=1-\left(\frac{V}{U_{\infty}}\right)^{2}$

From Equation (7), we can derive the relation between the pressure coefficient and the cavitation number on the cavity surface:

$-C_{P}=\frac{p_{\infty}-p}{1 / 2 \rho U_{\infty}^{2}}>\frac{p_{\infty}-p_{v}}{1 / 2 \rho U_{\infty}^{2}} \equiv \sigma$

Equation (8) implies that if the cavitation number $(\sigma)$ is smaller than the pressure coefficient $\left(-C_{p}\right)$, then cavitation occurs. If the pressure on the free streamline is equal to the ambient pressure, the length of the cavity is finite. It says the cavity length depends on the cavitation number $(\sigma)$. Cavity length is obtained through an iteration process in the boundary value problem. We, here, assume that the cavity length and compute the pressure along the initially guessed cavity surface until the computed cavitation number is equal to that of Equation (8).

\section{DISCRETIZATION OF SINGULARITIES}

In a boundary value problem, velocity potentials induced by source and dipole distributions are expressed as follow:

$\phi_{S}=\int \frac{q(s)}{2 \pi} \log r d s$
$\phi_{D}=\int \frac{\mu(s)}{2 \pi} \frac{\partial \log r}{\partial n} d s$

where $r=x-\xi$ is the distance between the field and singular point and $q(\xi), \mu(\xi)$ are strengths of distributed source and source respectively.

Using Green's $1^{\text {st }}$ and $2^{\text {nd }}$ theorems, the equation governing the internal flow can be expressed as an integral equation as follow: distribute dipoles on both body and cavity surfaces and also distributions of the sources on the cavity surface.

$\phi_{x}=U_{\infty} \cdot x+\int_{S_{B}+S_{C}} \frac{\mu(s)}{2 \pi} \frac{\partial \log r}{\partial n} d s+\int_{S_{C}} \frac{q(s)}{2 \pi} \log r d s$

where $S_{B}, S_{C}$ denote the body and cavity surface respectively.

The normal velocity component of the induced tangential velocity $(v)$ can be expressed in terms of the product of the oncoming velocity and the first derivative of cavity thickness:

$v=U_{\infty} \frac{d t_{c}}{d s}$

Integrating Equation (12) yields the cavity thickness and we adopt it as an alternative form of the cavity closure condition, which states that

$\left.t_{c}(l)\right)=\int_{0}^{s} \frac{q(s)}{U_{\infty}} d s=0$

We calculate the integral equation, Equation (11), along the discretized surfaces on which sources and dipoles are distributed:

$$
\begin{aligned}
\phi_{i}=U_{\infty} \cdot x_{i} & +\sum_{j=1}^{N_{D}} \frac{\mu_{j}}{2 \pi} \int_{p_{j}} \frac{\partial \log r}{\partial n} d s \\
& +\sum_{k=1}^{N_{S}} \frac{q_{k}}{2 \pi} \int_{p_{k}} \log r d s
\end{aligned}
$$

$i=1,2,3, \cdots, N_{D}$

where $N_{D}, N_{S}$ indicate the number of dipole and source panels $\left(p_{j}, p_{k}\right)$. 
We assume that the strengths of sources and dipoles are constant along each panel. We apply the detachment condition of the cavity at the trailing edge of the body, which states that the curvature of a free stream line at the starting point of the cavity is equal to the slope of the body. Consequently the source strength just behind the body can be calculated, and then Equation (14) is expressed in the form of applying the above formulation to the symmetric body, the number of singularity panels of unknown strengths is reduced by half.

$0=\phi_{i}=U_{\infty} \cdot x_{i}+\sum_{j=1}^{N_{D}} \frac{\mu_{j}}{2 \pi} \int_{p_{j}} \frac{\partial \log r}{\partial n} d s$

$+\frac{q_{1}}{2 \pi} \int_{p_{1}} \log r d s+\frac{q_{N_{S}}}{2 \pi} \int_{p_{N_{S}}} \log r d s+\sum_{k=2}^{N_{S}-1} \frac{q_{k}}{2 \pi} \int_{p_{k}} \log r d s$

As strengths of normal dipoles and sources are found, a new function for the cavity thickness is calculated from Equation (13), and it is added in the normal direction to the previous cavity surface. Singularities are placed into the newly generated surfaces and this process is conducted iteratively until the convergence criterion is satisfied.

\section{EXPERIMENTAL OBSERVARIONS}

Cavitation generated on a symmetric wedge has been investigated throughout experimental observations carried in a high speed cavitation tunnel at Chungnam National University, CNU CT. Fig. 2 shows the tunnel and its specification is shown in Table 1.
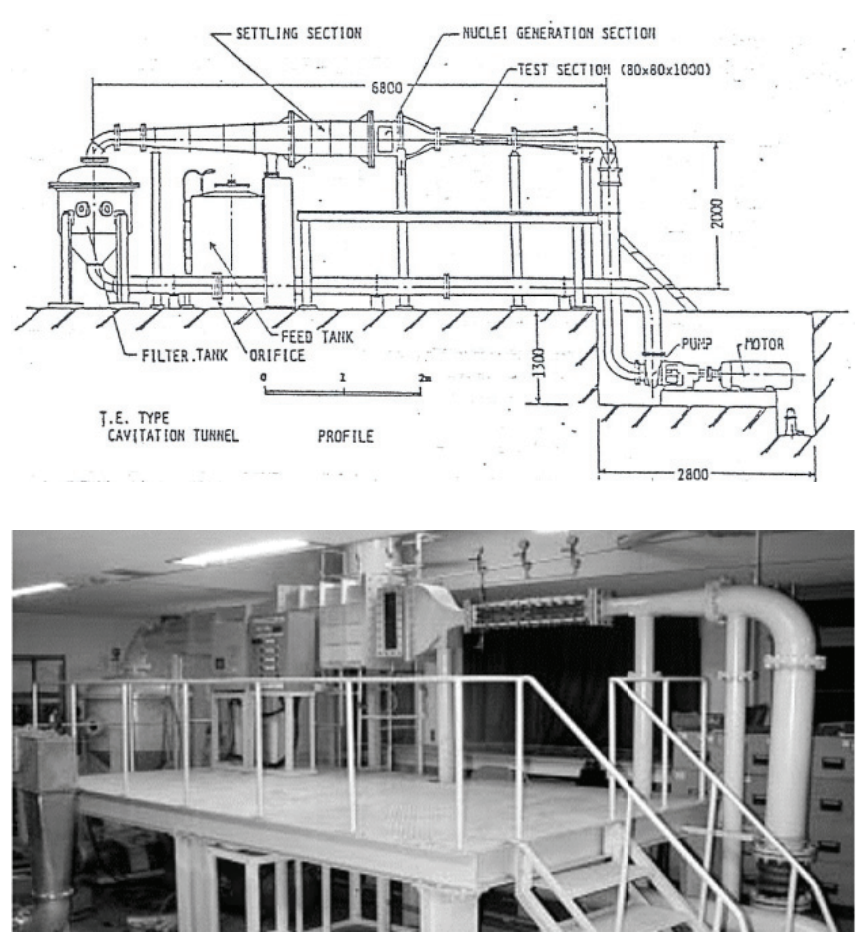

Fig. 2 CNU high speed cavitation tunnel
Table 1 Specification of the cavitation tunnel.

\begin{tabular}{|c|c|c|c|}
\hline $\begin{array}{c}\text { Tunnel } \\
(\mathrm{L} \times \mathrm{H})\end{array}$ & $6.8 m \times 2.0 \mathrm{~m}$ & $\begin{array}{c}\text { Speed } \\
(\max )\end{array}$ & $15 \mathrm{~m} / \mathrm{s}$ \\
\hline $\begin{array}{c}\text { Test section } \\
(\mathrm{D} \times \mathrm{B})\end{array}$ & $120 \mathrm{~mm} \times 50 \mathrm{~mm}$ & $\begin{array}{c}\text { Cav. No. } \\
\text { Range }\end{array}$ & $0.4 \sim 5.0$ \\
\hline $\begin{array}{c}\text { Pressure } \\
(\mathrm{min})\end{array}$ & $10 \mathrm{kPa}$ & $\begin{array}{c}\text { Pressure } \\
(\max )\end{array}$ & $300 \mathrm{kPa}$ \\
\hline
\end{tabular}

Fig. 3 shows the test section in where a wedge $(68.7 \mathrm{~mm}$ length and $18.1 \mathrm{~mm}$ thickness) is placed.

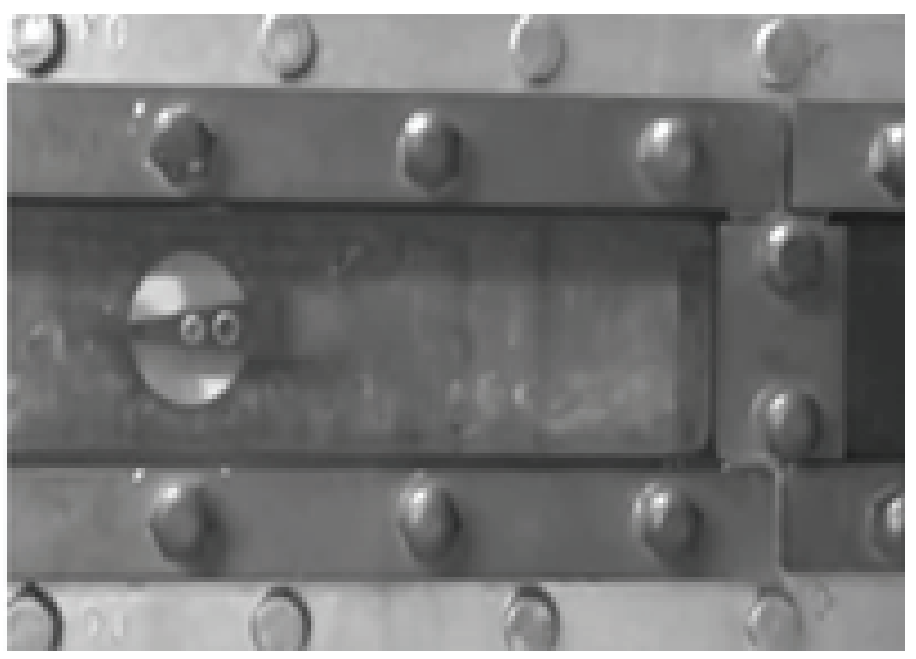

Fig. 3 Test section of the cavitation tunnel.

\section{RESULTS AND DISCUSSIONS}

Fig. 4 shows a typical result from the present numerical method. It gives that pressure and induced velocity distributions on both of body and cavity surfaces. One can read that the body having an angle of $15^{\circ}$ generates a cavity behind it of twice the wedge length at the cavitation number 0.27 .

Predicted cavity length is compared with an analytic solution in Fig. 5. Newman (1997) derived the relation between the cavitation number $(\sigma)$ and the cavity length $(l)$ for a two dimensional symmetric body:

$$
\frac{\pi}{4} \sigma l+\int_{0}^{1}\left(\frac{l+1}{t}\right)^{1 / 2} y_{0}^{\prime}(t) d t=0
$$

where $y_{0}(t)$ is the half thickness of the body. Present results are in good agreement with analytic solution as the cavitation number decreases. 


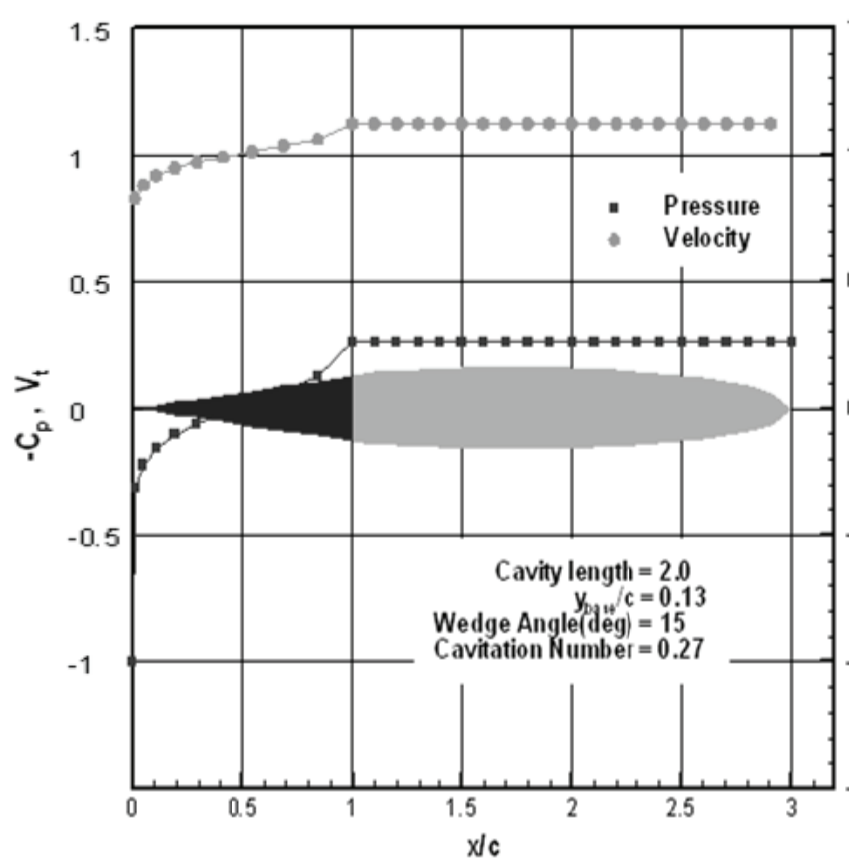

Fig. 4 Typical result of the present method: pressure, velocity distributions and super cavitation generated by a wedge at a certain cavitation number.

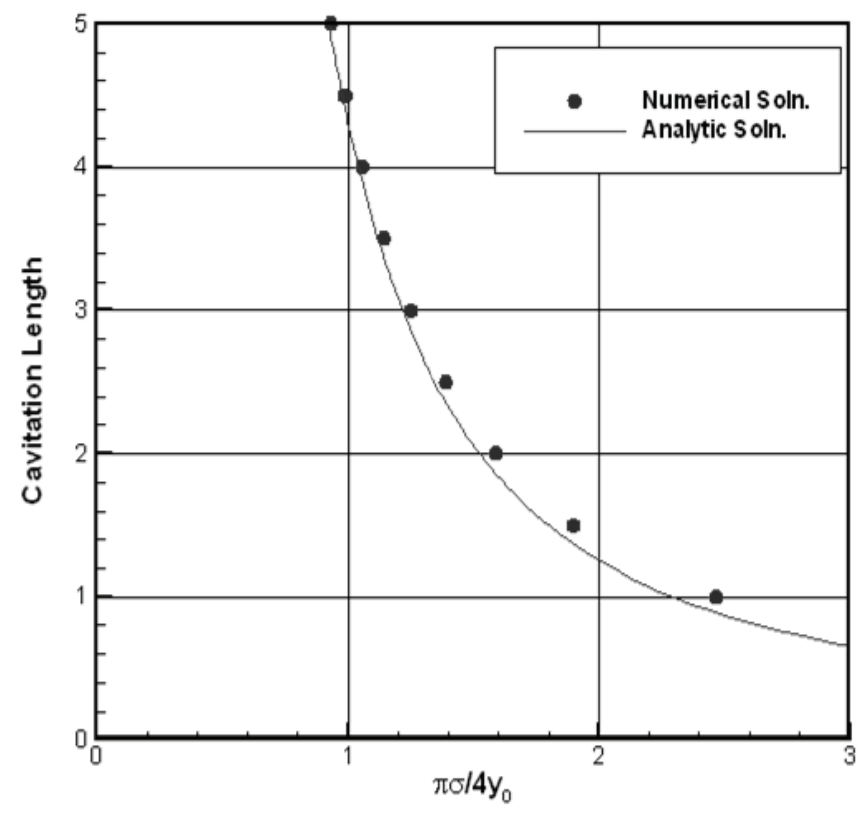

Fig. 5 Comparison of the cavity lengths between analytic and numerical solutions for a wedge described in Fig. 4.

Fig. 6 shows predicted cavities for different wedge shapes, relatively thin and blunt: (a) is for $45^{\circ}$ and (b) is for $90^{\circ}$ wedge angles. Both cases give the same cavity length, 9 and 10 times as long as the body. However, it is clearly shown that the thin wedge (smaller angle) needs low cavitation number to guarantee the similar length of the cavity comparing with the blunt wedge.

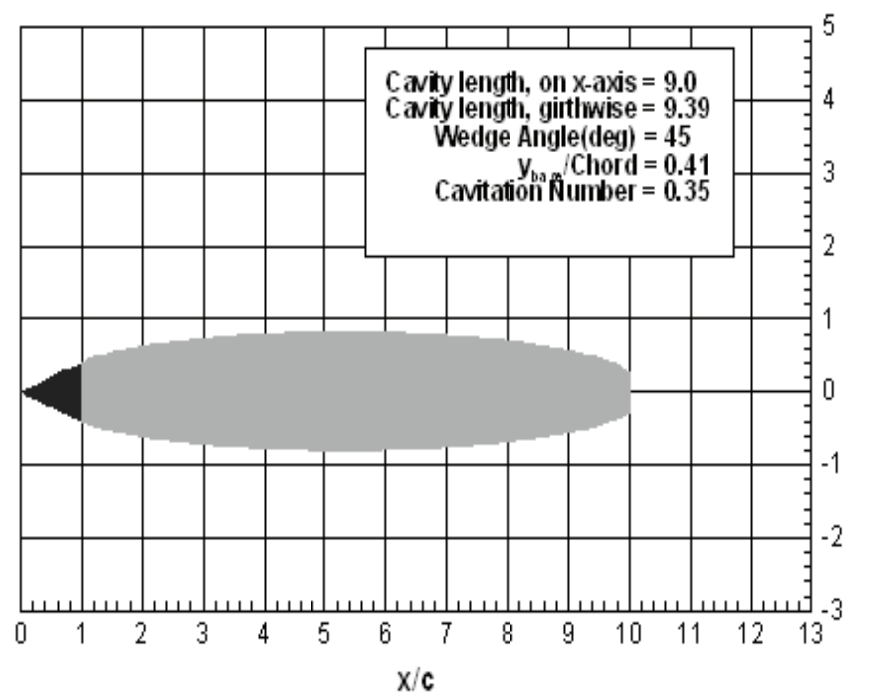

(a) wedge angle: $45^{\circ}$

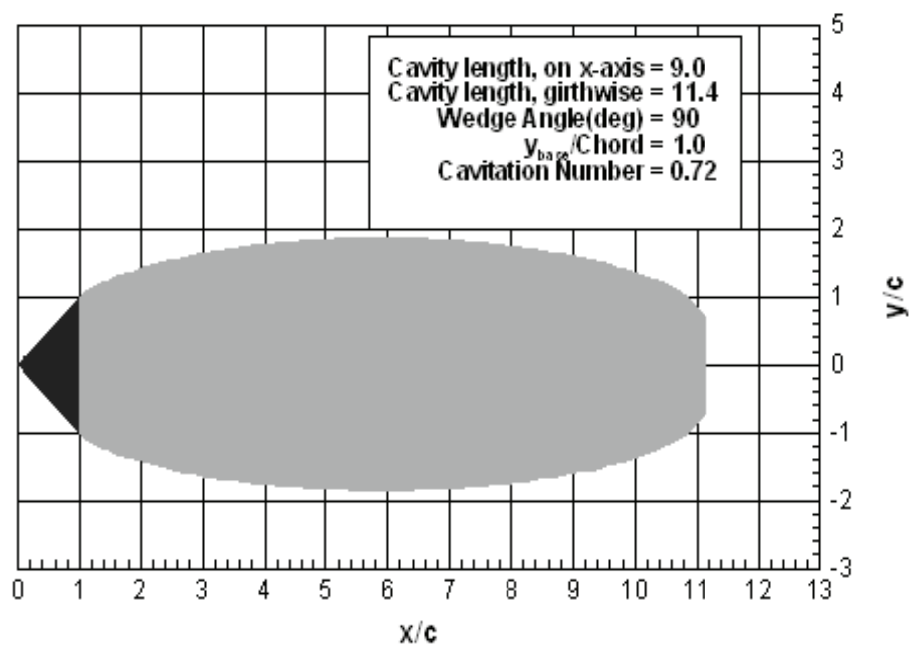

(b) wedge angle: $90^{\circ}$.

Fig. 6 Predicted cavity shapes for different bodies.

Left column of Fig. 7 shows growth of the cavity from the wedge sequentially. As the cavitation number decreases from 0.67 to 0.23 , cavity length increases from 0.5 to 2.5 times as long as the body. Right column of Fig. 7 shows the observed cavities at the same cavitation numbers as with the predictions. It was difficult to measure the end of the cavity apparently in this experimental trial. Comparing between them, it shows that the present method predicts well the cavity shape and its length as well. We are planning to record the cavity growth with the high-speed camera and also to figure. out the best way to measure the cavity length exactly.

Fig. 8 shows the cavity of the axisymmetric body which has $45^{\circ}$ corn angle. Here the cavitation number is 0.35 . We are now carrying out the experimental observations for the same body and it will be presented in the conference. 

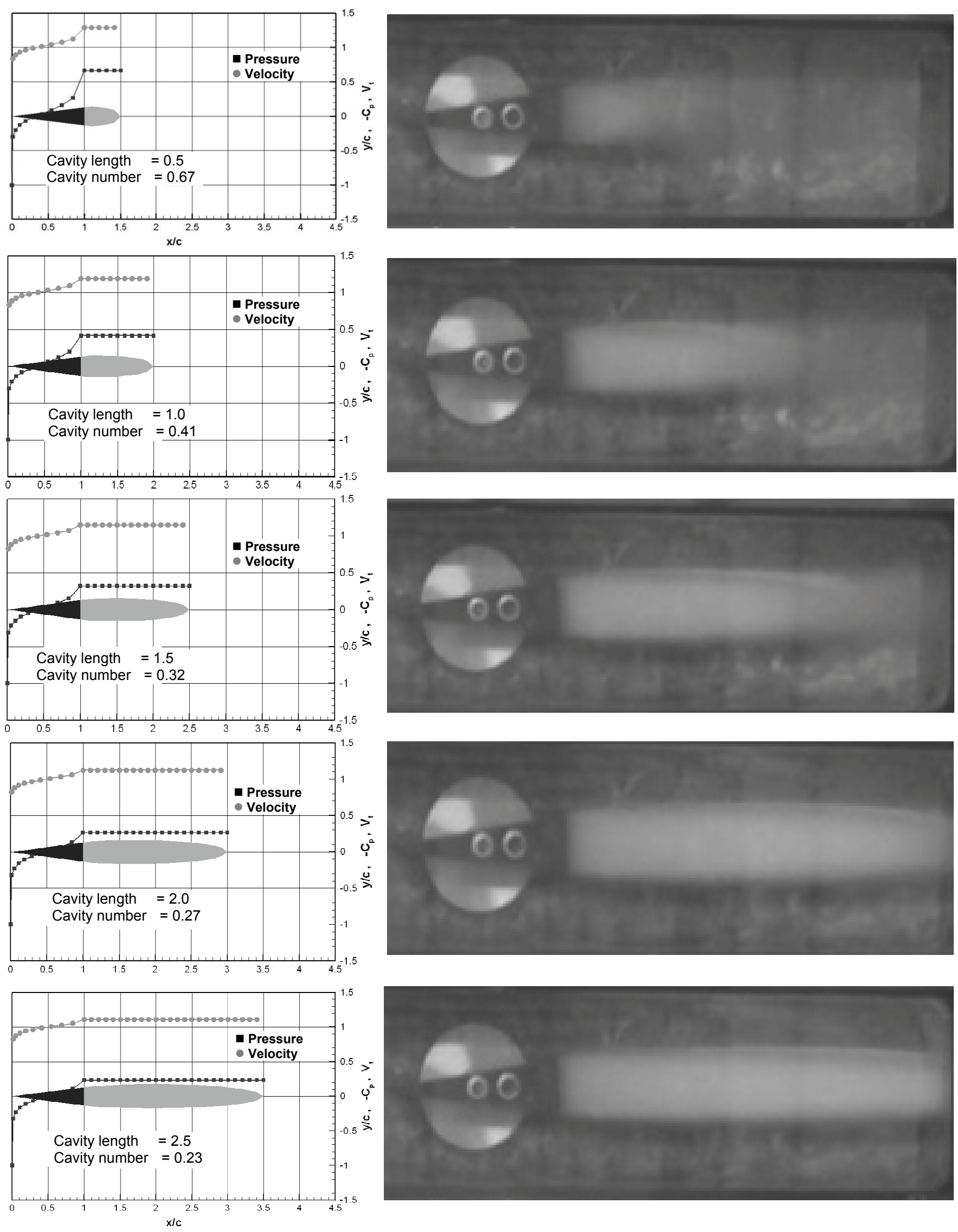

Fig. 7 Predicted (left column) and observed (right column) cavity growths as the cavitation number decreases from 0.67 to 0.23. 


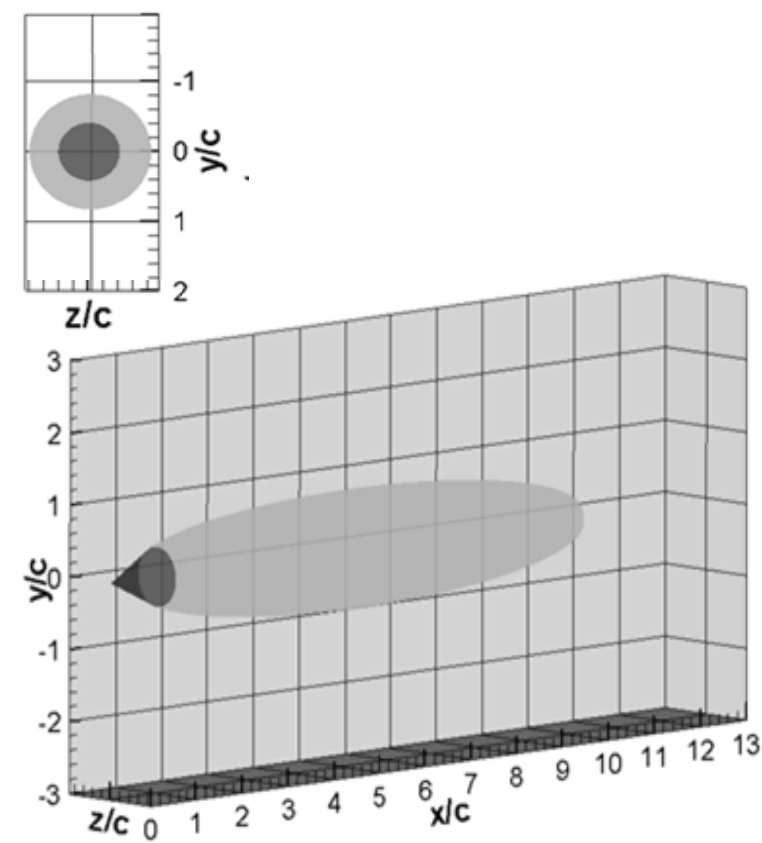

Fig. 8 Predicted cavity for axisymmetric body (corn angle is $45^{\circ}$ ) at $\sigma=0.35$.

\section{CONCLUSIONS}

In this study, a numerical method to predict supercavitation behind symmetric bodies is presented. Experimental observations are carried out in a high speed cavitation tunnel. The results show that the present method is capable of predicting the cavity shape and its length as well. At the design stage of the underwater supercavitating body, it is important to find a cavitator ensuring the sufficient cavity length covering the body. Consequently, the present method can be a useful design tool of the super cavitator.

\section{ACKNOWLEDGEMENTS}

This work was carried out with the support of Agency for Defense Development (ADD) under Grand No. 09-01-05-26.

\section{REFERENCES}

Alyanak, E. Venkayya, V. Grandhi, R. and Penmetsa, R., 2004. Variable shape cavitator design for a supercavitating torpedo. Proc. of $10^{\text {th }}$ AIAA/ISSMO Multidisciplinary Analysis and Optimization Conference, Albany, NY, USA.

Kirschner, I. N. Uhlman, J.S. Varghese, A.N. and Kuria, I.M., 1995. Supercavitating projectiles in axisymmetric subsonic liquid flows. American Society of Mechanical Engineers, Fluids Engineering Division, Vol. 210, pp. 75-93.

Kreisel, G. 1946. Cavitation with Finite Cavitatin Number, Admiralty Res. Lab. Rept. No. R1H/36.

Kunz, R.F. Boger, D.A. Chyczewski, T.S. Stinebring, D.R Gilbeling, H.J. and Govindan, T.R., 1999. Multiphase CFD analysis of natural and ventilated cavitation about submerged bodies. Proceeding of FEDSM 9, $3^{\text {rd }}$ ASME/JSME Joint Fluids Engineering Conference, San Francisco, CA, USA.

Lee, C.-S., 1989. A Potential-base panel method for the analysis of a 2-dimensional cavitating hydrofoil, College of Engineering, CNU, Deajon, Korea.

Newman, J.N., 1977. Marine Hydrodynamics, The MIT Press, Cambridge, MA, USA.

Uhlman, J.S., 1989. The Surface singularity or boundary integral method applied to supercavitating hydrofoils. J. of Ship Research, 33, pp. 16-20.

Wu, T.Y., 1972. Cavity and wake flow. Annual Review of Fluid Mechanics. 4, pp. 243-284.

Yang, C. and Jesup, S., 1988. Benchmark analysis of a series of propellers with a panel method. Proc. of SNAME propeller 1988 Symp., Virginia Beach, VA, USA, pp. 1-10. 